OPEN ACCESS

Edited by:

Nadine Ravel,

INSERM U1028 Centre de

Recherche en Neurosciences de

Lyon, France

Reviewed by:

Silvia Mandillo,

Institute of Cell Biology and

Neurobiology, CNR, Italy

Lionel Dahan,

Université Toulouse III Paul Sabatier,

France

*Correspondence:

Koshi Murata

kmurata@u-fukui.ac.jp

Received: 22 October 2018

Accepted: 27 February 2019

Published: 15 March 2019

Citation:

Murata K, Kinoshita T, Fukazawa Y, Kobayashi K, Yamanaka A, Hikida T,

Manabe $H$ and Yamaguchi $M$

(2019) Opposing Roles of Dopamine

Receptor D1- and D2-Expressing

Neurons in the Anteromedial

Olfactory Tubercle in Acquisition of

Place Preference in Mice.

Front. Behav. Neurosci. 13:50.

doi: 10.3389/fnbeh.2019.00050

\section{Opposing Roles of Dopamine Receptor D1- and D2-Expressing Neurons in the Anteromedial Olfactory Tubercle in Acquisition of Place Preference in Mice}

\author{
Koshi Murata ${ }^{1,2 *}$, Tomoki Kinoshita ${ }^{1}$, Yugo Fukazawa ${ }^{1,2,3}$, Kenta Kobayashi ${ }^{4}$, \\ Akihiro Yamanaka ${ }^{5}$, Takatoshi Hikida ${ }^{6}$, Hiroyuki Manabe ${ }^{7}$ and Masahiro Yamaguchi ${ }^{8}$ \\ ${ }^{1}$ Division of Brain Structure and Function, Faculty of Medical Sciences, University of Fukui, Fukui, Japan, ${ }^{2}$ Life Science \\ Innovation Center, Faculty of Medical Science, University of Fukui, Fukui, Japan, ${ }^{3}$ Research Center for Child Mental Health \\ Development, Faculty of Medical Sciences, University of Fukui, Fukui, Japan, ${ }^{4}$ Section of Viral Vector Development, National \\ Institute for Physiological Sciences, Aichi, Japan, ${ }^{5}$ Department of Neuroscience II, Research Institute of Environmental \\ Medicine, Nagoya University, Aichi, Japan, ${ }^{\circ}$ Laboratory for Advanced Brain Functions, Institute for Protein Research, Osaka \\ University, Osaka, Japan, 'Laboratory of Neural Information, Graduate School of Brain Science, Doshisha University, Kyoto, \\ Japan, ${ }^{8}$ Department of Physiology, Kochi Medical School, Kochi University, Kochi, Japan
}

Olfaction induces adaptive motivated behaviors. Odors associated with food induce attractive behavior, whereas those associated with dangers induce aversive behavior. We previously reported that learned odor-induced attractive and aversive behaviors accompany activation of the olfactory tubercle (OT) in a domain- and cell type-specific manner. Odor cues associated with a sugar reward induced attractive behavior and c-fos expression in the dopamine receptor D1-expressing neurons (D1 neurons) in the anteromedial OT. In contrast, odor cues associated with electrical shock induced aversive behavior and c-fos expression in the pamine receptor D2-expressing neurons (D2 neurons) in the anteromedial OT, as well as the D1 neurons in the lateral OT. Here, we investigated whether the D1 and D2 neurons in the anteromedial OT play distinct roles in attractive or aversive behaviors, using optogenetic stimulation and real-time place preference (RTPP) tests. Mice expressing ChETA (ChR2/E123T)-enhanced yellow fluorescent protein (EYFP) in the D1 neurons in the anteromedial OT spent a longer time in the photo-stimulation side of the place preference chamber than the control mice expressing EYFP. On the other hand, upon optogenetic stimulation of the D2 neurons in the anteromedial OT, the mice spent a shorter time in the photo-stimulation side than the control mice. Local neural activation in the anteromedial OT during the RTPP tests was confirmed by c-fos mRNA expression. These results suggest that the D1 and D2 neurons in the anteromedial OT play opposing roles in attractive and aversive behaviors, respectively.

Keywords: olfactory tubercle, attractive behavior, aversive behavior, place preference, optogenetics, medium spiny neurons, dopamine receptor D1, dopamine receptor D2 


\section{INTRODUCTION}

Odor sensation elicits various motivations, which enable adaptive behavioral responses such as obtaining food rewards or avoiding potential dangers (Doty, 1986). Although some odorants elicit innate motivated behaviors in mice, such as fear responses to predator odors (Kobayakawa et al., 2007; Saito et al., 2017) or attractive responses to social odors (Inokuchi et al., 2017), animals can acquire appropriate behaviors to odor cues according to their experience, through odor-reward or odor-danger associative learning. However, the neural circuit mechanisms engaged in these odor-induced adaptive behaviors are still unclear.

Recent studies have revealed the importance of the olfactory tubercle (OT) in the odor-induced motivated behaviors (DiBenedictis et al., 2015; Gadziola et al., 2015; Yamaguchi, 2017; Zhang et al., 2017a; Murofushi et al., 2018). The OT is a part of the olfactory cortex that receives olfactory inputs directly from the olfactory bulb as well as indirectly from other parts of the olfactory cortex and the orbitofrontal cortex (Shepherd, 2004; Zhang et al., 2017b). The OT is also a part of the ventral striatum, in addition to the nucleus accumbens (NAc), which receives massive dopaminergic inputs from the ventral tegmental area (Ikemoto, 2007; Park et al., 2017; Zhang et al., 2017b; Poulin et al., 2018). The OT is composed of three major types of neurons: medium spiny neurons, dwarf cells, and granule cells (Millhouse and Heimer, 1984; Xiong and Wesson, 2016). The medium spiny neurons are distributed in the whole OT, forming the layer II (dense cell layer) of the cortex-like region (Millhouse and Heimer, 1984). A majority of the medium spiny neurons in the OT as well as the NAc and dorsal striatum express either dopamine receptor D1 or D2 (Yung et al., 1995; Murata et al., 2015). Dwarf cells are clustered in the lateral part of the OT, forming the cap region, which is interspersed throughout the antero-posterior axis (Hosoya and Hirata, 1974; Murata et al., 2015). The dwarf cells are considered a smaller type of the medium spiny neurons, and express D1 but not D2 (Murata et al., 2015). Granule cells are clustered through the anteromedial surface to the central deep part of the OT, forming the Islands of Calleja, which is presumably a continuous structure (Fallon et al., 1978; de Vente et al., 2001). The granule cells weakly express D1, and do not express D2 (Murata et al., 2015). In addition to these three types of neurons in the striatal component, the OT contains the ventral pallidal component and axon bundles that project from the striato-pallidal structure to other brain areas, forming the medial forebrain bundle (Heimer, 1978).

In our previous study, we divided the OT into domains, using the cap and Islands of Calleja as a landmark, and mapped c-fos expression when mice showed learned odor-induced attractive or aversive behaviors (Murata et al., 2015). Odor cues associated with a sugar reward induced attractive behavior and c-fos expression in the D1-expressing neurons (D1 neurons) in the cortex-like region of the anteromedial domain, which is covered by the superficially located Islands of Calleja. In contrast, odor cues associated with electrical shock induced aversive behavior and c-fos expression in the D2-expressing neurons (D2 neurons) in the cortex-like region of the anteromedial domain, as well as D1 neurons in the cap and cortex-like regions of the lateral domain, which is surrounded by the cap region. These results raise the possibility that the D1 and D2 neurons in the anteromedial OT play opposing roles in odor-guided motivated behaviors. Consistent with this idea, the D1 and D2 neurons in the NAc have distinct roles in attractive and aversive learning (Hikida et al., 2010). Here, we investigated whether activation of the D1 and D2 neurons induces attractive and aversive behaviors, respectively, by combining optogenetic stimulation and real-time place preference (RTPP) tests (Zhang et al., 2017a).

\section{MATERIALS AND METHODS}

\section{Animals}

All experiments were conducted in accordance with the Guidelines for Animal Experimentation in Neuroscience of the Japan Neuroscience Society, and were approved by the Experimental Animal Research Committee of University of Fukui. The D1-Cre and D2-Cre mice used were heterozygotes and bred from D1-Cre (the Mutant Mouse Resource and Research Centers, STOCK Tg(Drd1a-cre)FK150Gsat/Mmucd, stock number: 029178-UCD; Gong et al., 2003, 2007) and D2-Cre mice (the Mutant Mouse Resource and Research Centers, B6.FVB(Cg)-Tg(Drd2-cre)ER44Gsat/Mmucd, stock number: 032108-UCD; Gong et al., 2003, 2007), by mating the heterozygote transgenic mice with wild-type C57BL/6J mice (Japan SLC, Inc., Shizuoka, Japan). Experiments were performed using male mice. After weaning, male mice were housed with their male littermates (2-6 mice per cage, wild-type and heterozygous mice were housed together) until the surgery, and then individually housed with a 12/12-h light/dark cycle. Food and water were freely available.

\section{Genotyping}

Genotyping of D1-Cre and D2-Cre mice were performed using conventional PCR with the following primers: D1-Cre, GCTATG GAGATGCTCCTGATGGAA-CGGCAAACGGACAGAAGC ATT (transgenic, 340-bp band; wild-type, no band); D2-Cre, GT GCGTCAGCATTTGGAGCAA-CGGCAAACGGACAGAAG CATT (transgenic, 700-bp band; wild-type, no band).

\section{Virus Preparation}

We used a Cre-dependent adeno-associated virus (AAV) vector encoding enhanced yellow fluorescent protein (EYFP) or ChETA(ChR2/E123T)-EYFP for cell type-specific gene expression. We obtained AAV5-EF1a-DIO-EYFP from the UNC vector core (Chapel Hill, NC, USA) at a titer of $3.5 \times 10^{12}$ genome copies/mL; AAV2-EF1a-DIO-ChETA-EYFP was packaged and concentrated to a titer of $1.6 \times 10^{12}$ genome copies/mL, as previously reported (Kobayashi et al., 2016), using the Addgene (Cambridge, MA, USA) plasmid, pAAV-EflaDIO ChETA-EYFP [gift from Dr. Karl Deisseroth, Stanford University, Stanford, CA, USA; \# 26968 (Gunaydin et al., 2010)].

\section{Stereotaxic Surgery}

Stereotaxic surgeries were performed on mice aged 10-16 weeks. Mice were anesthetized with a mixture of three anesthetics 
(medetomidine, midazolam, and butorphanol; Nakamura et al., 2017), and then placed in a stereotaxic apparatus (SR-5M; Narishige Co., Ltd., Tokyo, Japan). The skull above the targeted areas was thinned using a dental drill and removed carefully. The AAVs were injected using a syringe pump (UltraMicroPump III; World Precision Instruments, LLC, Sarasota, FL, USA) connected to a Hamilton syringe (RN-1701; Hamilton, Reno, $\mathrm{NV}$, USA), and a mounted glass micropipette with a tip diameter of $50 \mu \mathrm{m}$ connected by an adaptor (55750-01, Hamilton, Reno, NV, USA).

We unilaterally injected $300 \mathrm{~nL}$ AAV5-EF1a-DIO-EYFP for confirmation of cell type-specific expression (Figure 1A) and as a control for optogenetic stimulation, or AAV2-EF1a-DIOChETA-EYFP into the left hemisphere of the anteromedial OT of D1-Cre or D2-Cre mice using the following coordinates: anterior-posterior, $+1.5 \mathrm{~mm}$; medial-lateral, $0.7 \mathrm{~mm}$ from the bregma; and dorso-ventral, $4.35 \mathrm{~mm}$ from the brain surface. Two to three weeks later, the mice were ipsilaterally implanted with a chronic optical fiber (numerical aperture $=0.39,200-\mu \mathrm{m}$ diameter; CFMC12U; Thorlabs, Inc., Newton, NJ, USA) targeted to the anteromedial OT with the same coordinates described above. One to two weeks after fiber implantation, the following behavioral tests were conducted.

\section{Optogenetic Stimulation and RTPP Tests}

For optogenetic stimulation, the implanted optic fiber was connected to a blue light laser via patch cords with a fiberoptic rotary joint (RJPSF2; Thorlabs, Inc., Newton, NJ, USA). All photo-stimulation experiments used 5-ms, 5-7-mW, 473-nm light pulses at $20 \mathrm{~Hz}$ via a solid-state laser for light delivery (CSTL-473-50-OEM; Ultralasers, Inc., Newmarket, ON, Canada) triggered by a stimulator (STO2; Bio Research Center Co., Ltd., Nagoya, Japan). The intensity of the light-stimulation was verified by c-fos expression (Figure 4).

After being connected to the blue light laser, the mice were placed in a place preference chamber [30 (width) $\times 30$ (depth) $\times 25$ (height) $\mathrm{cm}^{3}$ ] equipped with vertical or horizontal striped wall, as shown in Figure 1B, for $20 \mathrm{~min}$. The non-stimulation control side was as assigned at the start of the experiment. Laser stimulation at $20 \mathrm{~Hz}$ was initiated when the mice entered the stimulation side, constantly delivered when the mice were in the stimulation side, and stopped when they returned back to the initial non-stimulation side. All behavioral tests were recorded using a USB digital video camera (Logicool c920r; Logitech, Lausanne, Switzerland). Offline analyzes of the time spent in each chamber, tracking data, and total traveled distance were performed using a video-tracking software (SMART 3.0; Panlab, Barcelona, Spain). Thirty minutes after the end of the RTPP tests, mice were deeply anesthetized by intraperitoneal injection of sodium pentobarbital, and then fixed for histochemical analysis.

\section{Histochemistry}

Mice were transcardially perfused with phosphate-buffered saline (PBS), followed by $4 \%$ paraformaldehyde (PFA). After cryoprotection with sucrose solution, the brain was frozen and sliced into coronal sections with a thickness of $20 \mu \mathrm{m}$.
The sections were rinsed in PBS and 0.1 M phosphate buffer, mounted on glass slides using a paint brush, dried overnight in a vacuum desiccator, and then stored at $4^{\circ} \mathrm{C}$ until histochemistry.

To confirm cell type-specific EYFP expression, we performed double fluorescence labeling for EYFP and mRNAs of D1 or D2 as follows. Digoxigenin (DIG)-labeled RNA probes were prepared using an in vitro transcription kit (Roche, Basel, Switzerland) according to the manufacturer's protocol with a plasmid kindly provided by Dr. Kazuto Kobayashi (Sano et al., 2003). The dried sections were fixed in 4\% PFA, digested using proteinase $\mathrm{K}(10 \mu \mathrm{g} / \mathrm{mL})$ for $30 \mathrm{~min}$, and post-fixed in 4\% PFA. After prehybridization, the sections were incubated overnight at $65^{\circ} \mathrm{C}$ with DIG-labeled RNA probes. After stringent washing, the sections were incubated in 1\% blocking buffer (11096176001, Roche, Basel, Switzerland) for $1 \mathrm{~h}$. Primary antibody against EYFP (1:1,000; Medical and Biological Laboratories Co., Ltd., Nagoya, Japan) and an anti-DIG antibody conjugated with alkaline phosphatase (1:500, Roche, Basel, Switzerland) were included in the incubation mixture. The sections were washed three times in TNT [0.1 M Tris- $\mathrm{HCl}$ ( $\mathrm{pH} 7.5$ ), $0.15 \mathrm{M} \mathrm{NaCl}, 0.1 \%$ Tween 20] and incubated with an Alexa Fluor 488-conjugated secondary antibody (1:400; Jackson ImmunoResearch Labs, Inc., West Grove, PA, USA) for $2 \mathrm{~h}$. After three washes in TNT and one wash in Tris saline [0.1 M Tris- $\mathrm{HCl}(\mathrm{pH} 8.0), 0.1 \mathrm{M} \mathrm{NaCl}$, $50 \mathrm{mM} \mathrm{MgCl}_{2}$ ], alkaline phosphatase activity was detected using the HNPP Fluorescence Detection Set (11758888001, Roche, Basel, Switzerland) according to the manufacturer's instructions. The sections were incubated with the substrate three times for $30 \mathrm{~min}$ each, and the reaction was stopped by washing the sections in PBS. The sections were then counterstained with $4^{\prime}, 6$ diamidino-2-phenylindole diluted in PBS $(2 \mu \mathrm{g} / \mathrm{mL})$ for $5 \mathrm{~min}$. After washing in PBS, the sections were mounted in PermaFluor (Thermo Fisher Scientific, Waltham, MA, USA).

For c-fos mRNA detection, we performed in situ hybridization using DIG-labeled antisense RNA probes. The RNA probe was prepared using an in vitro transcription kit (Roche, Basel, Switzerland) according to the manufacturer's protocol with a plasmid kindly provided by Dr. Hirohide Takebayashi (Bepari et al., 2012). Hybridization and washing were performed as described above. Subsequently, the sections were blocked with $10 \%$ normal sheep serum, $1 \%$ bovine serum albumin, and $0.1 \%$ Triton X-100 in PBS. The sections were then incubated overnight at $4^{\circ} \mathrm{C}$ with alkaline phosphatase-conjugated anti-DIG antibody $(1: 1,000$, Roche, Basel, Switzerland). The sections were washed in TNT, followed by alkaline phosphatase buffer $[100 \mathrm{mM} \mathrm{NaCl}, 100 \mathrm{mM}$ Tris- $\mathrm{HCl}$ ( $\mathrm{pH}$ 9.5), $50 \mathrm{mM} \mathrm{MgCl}_{2}, 0.1 \%$ Tween $20,5 \mathrm{mM}$ levamisole]. The sections were treated overnight with nitro-blue tetrazolium/5-bromo-4-chloro-3' -indolylphosphate (Roche, Basel, Switzerland) mixture at room temperature in a dark room for color development. Subsequently, they were rinsed in PBS and mounted in PermaFluor (Thermo Fisher Scientific, Waltham, MA, USA).

\section{Microscopy and Image Analysis}

Sections were examined using a confocal laser microscope (FV1200, Olympus, Tokyo, Japan) and a bright-field virtual 
slide system (NanoZoomer; Hamamatsu Photonics, Shizuoka, Japan). To quantify the number of c-fos mRNA-expressing cells, three coronal sections of the anteromedial OT with $20 \mu \mathrm{m}$ thickness were selected (centering [antero-posterior axis] section and $100 \mu \mathrm{m}$-anterior and posterior sections). The number of cells in the anteromedial domain of the OT and NAc medial shell was counted using ImageJ (National Institutes of Health, Bethesda, MD, USA).

\section{Criteria for Data Analysis}

We confirmed that all the mice analyzed in the EYFP-expressing control group (shown in Figures 3, 4) expressed EYFP in the anteromedial OT and that the tip of the optic fiber was placed above the anteromedial OT. In the ChETA-EYFP group (shown in Figures 3, 4), the mice in which the center of the c-fos activation was located outside of the anteromedial OT (including the NAc) were excluded from data analysis [a total of 12 D1-Cre and D2-Cre mice (6 each) were excluded].

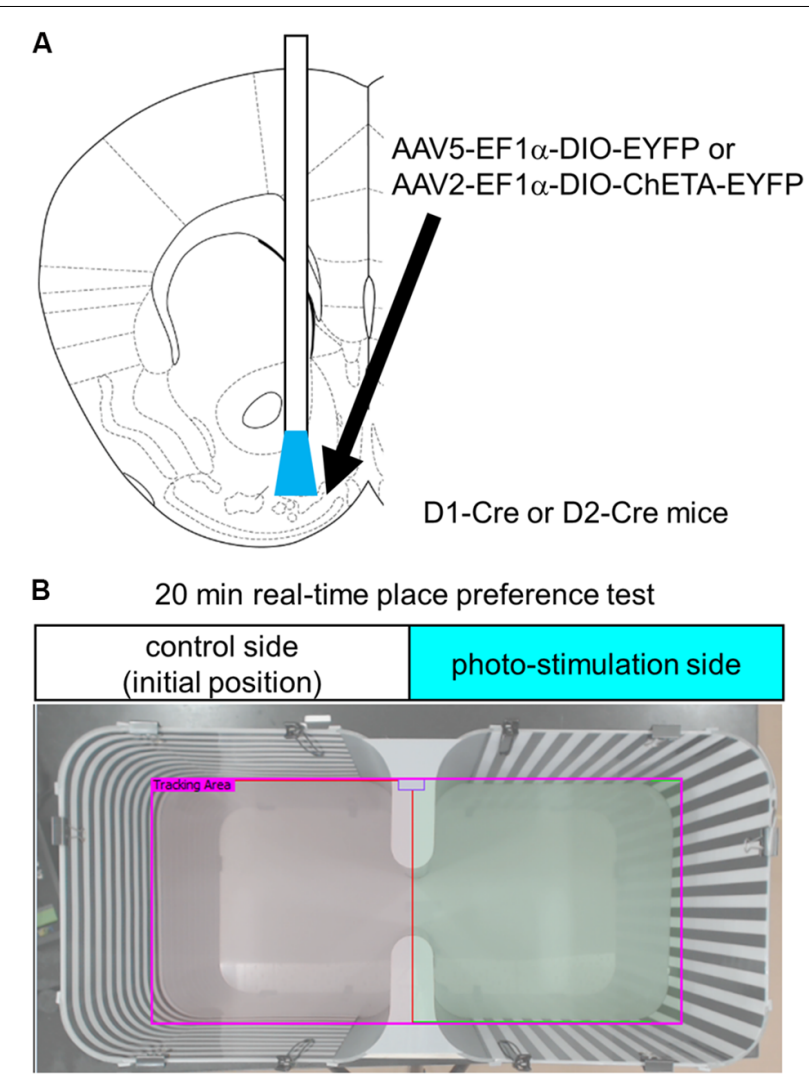

FIGURE 1 | Experimental design. (A) Schematic diagram of cell type-specific optogenetic stimulation of the anteromedial OT. Stereotaxic atlas from Franklin and Paxinos (2008). We injected Cre-dependent AAVs encoding EYFP or ChETA-EYFP and implanted optic fiber canula into the anteromedial OT of D1-Cre or D2-Cre mice. (B) Place preference chamber. In the RTPP tests, mice were randomly placed on either side of the chamber, which was assigned as the control side (no photo-stimulation). Blue light was delivered when mice were on the opposite side of the initial position. OT, olfactory tubercle; AAV, adeno-associated virus; EYFP, enhanced yellow fluorescent protein; RTPP, real-time place preference; D1, dopamine receptor D1; D2, dopamine receptor D2.

\section{Statistics}

Normality of the quantitative data presented in Figures 3, 4 was confirmed with the Kolmogorov-Smirnov test, using the $\mathrm{R}$ software (version 3.5.2; The $\mathrm{R}$ Foundation for Statistical
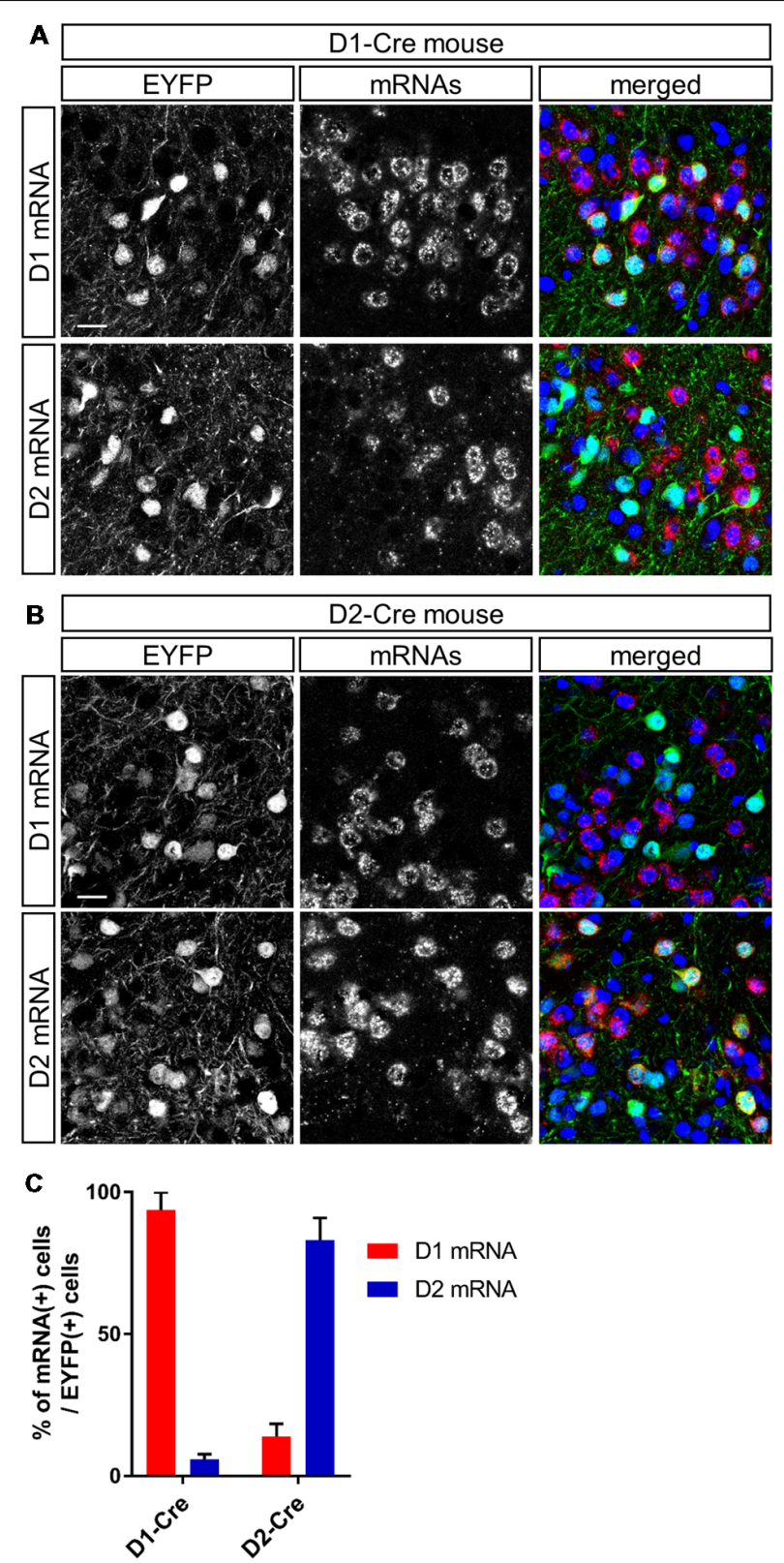

FIGURE 2 | Cell type-specific gene expression in dopamine receptor D1 and D2-expressing neurons in the anteromedial OT of the D1-Cre and D2-Cre mice using AAV vectors. (A,B) Confocal images of AAV-derived EYFP-expressing cells (green) and D1 (upper panels) or D2 (lower panels) mRNAs (red) from a D1-Cre (A) or D2-Cre (B) mouse. Color merged panel contains DAPI staining (blue). Scale bars: $20 \mu \mathrm{m}$. (C) Percentage of D1 or D2 mRNA-expressing cells among EYFP-expressing cells in D1-Cre and D2-Cre mice. Data show mean with SD. OT, olfactory tubercle; AAV, adeno-associated virus; EYFP, enhanced yellow fluorescent protein; D1, dopamine receptor D1; D2, dopamine receptor D2; DAPI,

4',6-diamidino-2-phenylindole; SD, standard deviation. 
Computing, Vienna, Austria). Statistical significance was tested with parametric tests [Figure 3; two-way analysis of variance (ANOVA) with post hoc Tukey's test, Figure 4; three-way ANOVA with post hoc Tukey's test], using the Prism 8 software (GraphPad Software, San Diego, CA, USA). Quantitative data are shown as mean \pm standard deviation or mean with individual data plots. Differences were considered statistically significant at $p<0.05$.

\section{RESULTS}

To address whether D1 and D2 neurons in the anteromedial OT play distinct roles in attractive and aversive behaviors, we used optogenetic stimulation and performed RTPP tests (Figures 1A,B). We injected AAV2-EF1a-DIO-ChETA-EYFP into the anteromedial OT of D1-Cre and D2-Cre transgenic mice; AAV5-EF1a-DIO-EYFP was injected as a control for optogenetic stimulation (Figure 1A). At first, we examined cell type-specificity of the Cre-mediated gene expression by the AAV vector. Three weeks after injection of AAV5-EF1aDIO-EYFP into the anteromedial OT of the D1-Cre and D2-Cre mice, we performed double fluorescence labeling of EYFP and D1 or D2 mRNAs (Figures 2A,B). In the D1-Cre mice, $93.7 \pm 6.2 \%$ of the $\operatorname{EYFP}(+)$ neurons in the cortex-like region, which were putative medium spiny neurons, expressed D1 mRNA, and $5.9 \pm 1.8 \%$ of them expressed D2 mRNA ( $n=3$ mice, Figures 2A,C). On the other hand, $14.0 \pm 4.3 \%$ of the $\operatorname{EYFP}(+)$ neurons expressed D1 mRNA, and approximately $83.1 \pm 7.8 \%$ of them expressed D2 mRNA in the D2-Cre mice ( $n=3$ mice, Figures $\mathbf{2 B}, \mathbf{C}$ ). These data confirmed that these Cre transgenic mice exhibited preferential expression of Cre-dependent AAV-derived genes in the D1 and D2 neurons.
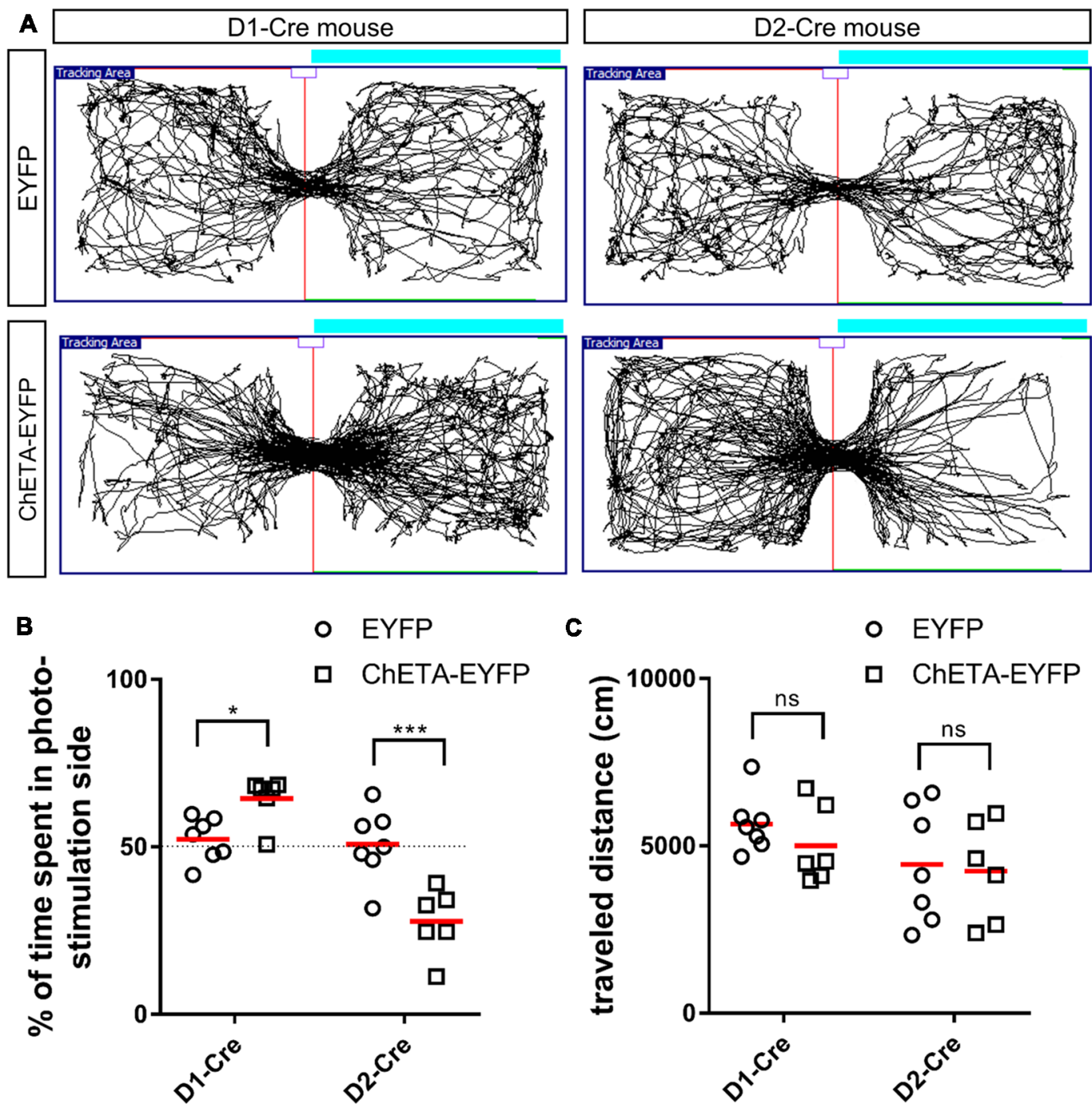

FIGURE 3 | Cell type-specific effect of optogenetic stimulation of the anteromedial OT in the RTPP tests. (A) Tracking data of the RTPP tests. Right side is the photo-stimulation side. (B) Percentage of time spent in the photo-stimulation side in the 20-min RTPP tests. (C) Traveled distance during the 20-min RTPP tests. Data show mean with individual plots. ns, not significant; * $p<0.05$; ${ }^{* * *} p<0.001$. OT, olfactory tubercle; RTPP, real-time place preference. 
We then tested the hypothesis that optogenetic activation of the D1 and D2 neurons in the anteromedial OT may play distinct roles in eliciting attractive and aversive behaviors using RTPP tests. We activated the D1 and D2 neurons using ChETA, a type of ChR2 with faster kinetics (Gunaydin et al., 2010), which possibly enabled precise timing of stimulation when mice crossed the chambers. The RTPP tests revealed that D1-Cre mice expressing ChETA spent significantly longer time in the photo-stimulation side $(64.5 \pm 6.3 \%$ of the 20 -min RTPP tests, $n=6$ mice) than the control mice $(52.3 \pm 6.1 \%$ of the 20 -min RTPP tests, $n=7$ mice; two-way ANOVA: EYFP/ChETA-EYFP $F_{(1,22)}=2.68, p=0.12 ; \mathrm{D} 1-\mathrm{Cre} / \mathrm{D} 2-\mathrm{Cre} F_{(1,22)}=38.72, p<0.0001$; interaction $F_{(1,22)}=31.26, p<0.0001$, with post hoc Tukey's test: $p=0.048,95 \%$ confidence interval $=0.09-24.6$ ), which expressed EYFP without ChETA (Figures 3A,B). In contrast, D2-Cre mice expressing ChETA spent significantly shorter time in the photo-stimulation side $(27.8 \pm 9.0 \%$ of the 20 -min RTPP tests, $n=6$ mice) than the control mice (50.8 $\pm 9.9 \%$ of the 20-min RTPP tests, $n=7$ mice; post hoc Tukey's test: $p=0.0002,95 \%$ confidence interval $=-34.8$ to -10.3 ; Figures $3 \mathbf{A}, \mathbf{B})$. These data suggest that activation of the D1 and D2 neurons in the anteromedial OT elicit attractive and aversive behaviors, respectively. We did not observe statistically significant difference in the traveled distance in the 20-min RTPP tests for either D1-Cre (control, 5,655 $\pm 796 \mathrm{~cm}$; ChETA, $5,003 \pm 1,067 \mathrm{~cm}$ ) or D2-Cre (control, 4,447 $\pm 1,612 \mathrm{~cm}$; ChETA,
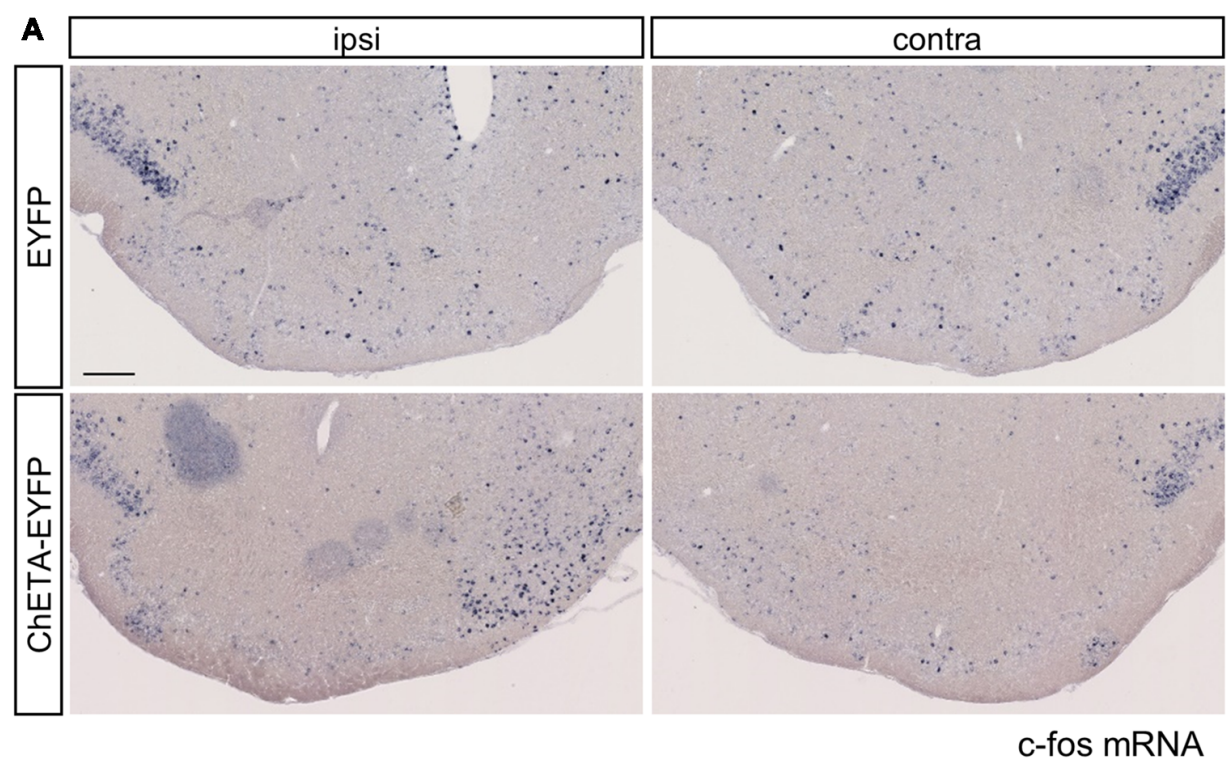

B

C
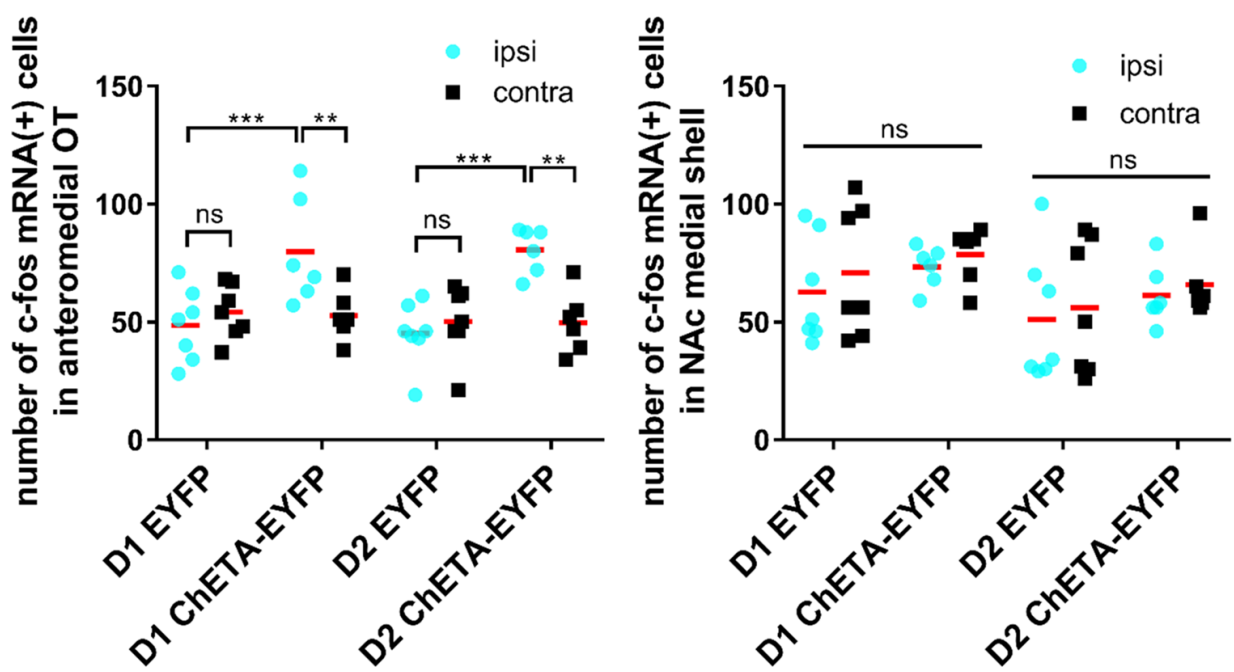

FIGURE 4 | c-fos expression induced by optogenetic stimulation was localized in the anteromedial OT during the RTPP tests. (A) Images of C-fos mRNA expression in the OT coronal sections after the RTPP tests. Left panels, ipsilateral; right panels, contralateral. Scale bar: $200 \mu \mathrm{m}$. (B) Number of c-fos mRNA-expressing cells in the anteromedial OT. (C) Number of c-fos mRNA-expressing cells in the NAc medial shell. Data shows mean with individual data plots. ns, not significant; ${ }^{* *} p<0.01 ;{ }^{* * *} p<0.001$. OT, olfactory tubercle; NAc, nucleus accumbens; RTPP, real-time place preference. 
$4,247 \pm 1,369 \mathrm{~cm}$ ) mice (two-way ANOVA: EYFP/ChETA-EYFP $F_{(1,22)}=0.63, p=0.43$; D1-Cre/D2-Cre $F_{(1,22)}=3.36, p=0.08$; interaction $F_{(1,22)}=0.18, p=0.68$; Figure $\left.3 \mathrm{C}\right)$.

After the RTPP tests, we confirmed the neural activation of the anteromedial OT by examining c-fos mRNA expression (Bepari et al., 2012). As expected, the ipsilateral side of the anteromedial OT in the ChETA-EYFP-expressing mice showed a significant increase in the number of c-fos-expressing cells $(80 \pm 21$ cells for D1-Cre, $81 \pm 9$ cells for D2-Cre) compared with both the contralateral side of the anteromedial OT in the ChETA-EYFP-expressing mice (53 \pm 10 cells for D1Cre, $50 \pm 12$ cells for D2-Cre) and the ipsilateral side in the control mice without ChETA ( $49 \pm 14$ cells for D1-Cre, $45 \pm 12$ cells for D2-Cre), for both D1-Cre (three-way ANOVA: EYFP/ChETA-EYFP, $F_{(1,22)}=10.02, p=0.005$; ipsi/contra, $F_{(1,22)}=22.86, p<0.0001 ;$ D1-Cre/D2-Cre, $F_{(1,22)}=0.23$, $p=0.64$; post hoc Tukey's test: vs. contralateral-ChETA-EYFP, $p=0.0006,95 \%$ confidence interval $=10-44$; vs. ipsilateralEYFP, $p=0.007,95 \%$ confidence interval $=6-57$ ) and D2-Cre (post hoc Tukey's test: vs. contralateral-ChETA-EYFP, $p=0.0001$, $95 \%$ confidence interval $=14-48$; vs. ipsilateral-EYFP, $p=0.002$, $95 \%$ confidence interval $=10-61$ ) mice (Figures $4 \mathbf{A}, \mathbf{B})$. The NAc did not show a significant increase in the number of c-fosexpressing cells in the either ChETA-EYFP-expressing D1-Cre or D2-Cre (three-way ANOVA: EYFP/ChETA-EYFP, $F_{(1,22)}=1.48$, $p=0.24$; ipsi/contra, $F_{(1,22)}=4.21, p=0.052$; D1-Cre/D2-Cre, $F_{(1,22)}=2.63, p=0.12$ ) mice (Figure $4 \mathrm{C}$ ). These results confirmed that the neural activation by optogenetic stimulation was specific to the anteromedial OT during the RTPP tests.

\section{DISCUSSION}

In this study, we demonstrate that cell type-specific activation of the D1 and D2 neurons in the anteromedial OT elicits attractive and aversive behaviors, respectively. To achieve selective manipulation of the D1 or D2 neurons, which are intermingled in the cortex-like region of the OT, we used D1-Cre and D2-Cre transgenic mouse lines and Cre-dependent AAV vectors (Figures 1A, 2). This combination enabled us to deliver AAV-derived genes preferentially to the D1 or D2 neurons in the anteromedial OT. Optogenetic activation of the D1 and D2 neurons in the anteromedial OT induced attraction to and aversion from the photo-stimulation chamber, respectively (Figures 1B, 3). The photo-stimulation did not induce significant changes in traveled distance during the RTPP tests, suggesting that locomotor activity was not significantly influenced by the optogenetic activation (Figure 3C). After the RTPP tests, activation of the anteromedial OT was confirmed by local increase in the $c$-fos expression (Figure 4). These results suggest that the D1 and D2 neurons in the anteromedial OT are involved in eliciting attractive and aversive behaviors, respectively (Figure 5).

Previous studies have shown that the anteromedial domain of the OT plays an important role in the reward system. Local self-administration of cocaine into the OT and NAc revealed that the anteromedial domain of the OT more robustly mediates the rewarding action of cocaine than other domains

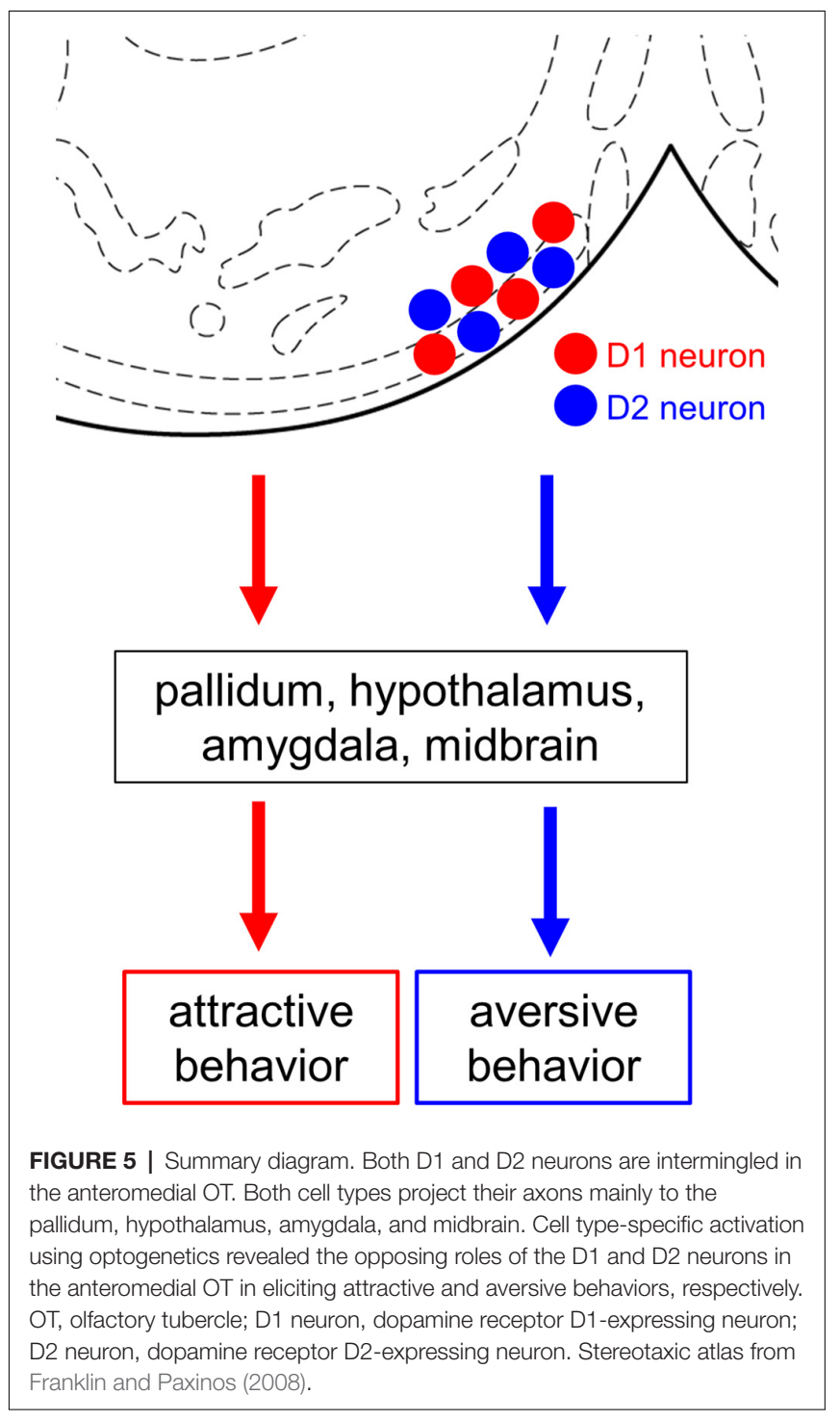

of the OT and NAc (Ikemoto, 2003). Optogenetic stimulation of the dopaminergic fiber from the ventral tegmental area to the medial OT elicits rewarding effects which generate place and odor preference (Zhang et al., 2017a). These local manipulations should exert excitatory effect on the D1 neurons via increased dopamine level in the anteromedial OT because D1 is coupled with Gs (Stoof and Kebabian, 1981). In line with these previous reports, our result directly demonstrates the role of the D1 neurons in the anteromedial OT in eliciting attractive behavior. In contrast, D2 is coupled with Gi (Stoof and Kebabian, 1981). Aversive stimuli reduce tonic firing of dopaminergic neurons, resulting in decreased ambient dopamine level at the target structure (Bromberg-Martin et al., 2010; Cohen et al., 2012; McCutcheon et al., 2012), which should exert excitatory effect on the D2 neurons. As blunting the tonic dopamine release in the ventromedial striatum leads to conditioned place aversion (Liu et al., 2008), our results support the idea that the D2 neurons in the anteromedial OT detect aversive stimuli via decreased dopamine release and elicit 
aversive behavior. Both D1 and D2 neurons in the medial OT project their GABAergic axons to mainly the reward-related brain regions, including the pallidum, hypothalamus, amygdala, and midbrain (Zhang et al., 2017b). Future neuroanatomical studies should address how the downstream neural circuits elicit attractive and aversive behaviors (Figure 5). As we previously reported, neurons in the OT are activated by odor cues that induce motivated behaviors. The understanding of these cell type-specific roles of the D1 and D2 neurons in the anteromedial OT will provide a neural basis for odor-guided adaptive motivated behaviors.

\section{AUTHOR CONTRIBUTIONS}

KM designed research, performed experiments, and wrote the manuscript. TK performed experiments. YF, KK, AY, TH, HM, and MY contributed tools and reagents, and assisted in revision of the manuscript.

\section{REFERENCES}

Bepari, A. K., Watanabe, K., Yamaguchi, M., Tamamaki, N., and Takebayashi, H. (2012). Visualization of odor-induced neuronal activity by immediate early gene expression. BMC Neurosci. 13:140. doi: 10.1186/1471-2202-13-140

Bromberg-Martin, E. S., Matsumoto, M., and Hikosaka, O. (2010). Dopamine in motivational control: rewarding, aversive, and alerting. Neuron 68, 815-834. doi: 10.1016/j.neuron.2010.11.022

Cohen, J. Y., Haesler, S., Vong, L., Lowell, B. B., and Uchida, N. (2012). Neurontype-specific signals for reward and punishment in the ventral tegmental area. Nature 482, 85-88. doi: 10.1038/nature10754

de Vente, J., Hani, L., Steinbusch, H. E., and Steinbusch, H. W. (2001). The three dimensional structure of the islands of Calleja: a single heterogenous cell complex. Neuroreport 12, 565-568. doi: 10.1097/00001756-200103050-00026

DiBenedictis, B. T., Olugbemi, A. O., Baum, M. J., and Cherry, J. A. (2015). DREADD-induced silencing of the medial olfactory tubercle disrupts the preference of female mice for opposite-sex chemosignals. eNeuro 2:ENEURO.0078-15.2015. doi: 10.1523/eneuro.0078-15.2015

Doty, R. L. (1986). Odor-guided behavior in mammals. Experientia 42, 257-271. doi: $10.1007 /$ bf01942506

Fallon, J. H., Riley, J. N., Sipe, J. C., and Moore, R. Y. (1978). The islands of Calleja: organization and connections. J. Comp. Neurol. 181, 375-395. doi: 10.1002/cne. 901810209

Franklin, K. B. J., and Paxinos, G. (2008). The Mouse Brain in Stereotaxic Coordinates. Compact 3rd Edn. San Diego, CA: Elsevier.

Gadziola, M. A., Tylicki, K. A., Christian, D. L., and Wesson, D. W. (2015). The olfactory tubercle encodes odor valence in behaving mice. J. Neurosci. 35, 4515-4527. doi: 10.1523/JNEUROSCI.4750-14.2015

Gong, S., Doughty, M., Harbaugh, C. R., Cummins, A., Hatten, M. E., Heintz, N., et al. (2007). Targeting Cre recombinase to specific neuron populations with bacterial artificial chromosome constructs. J. Neurosci. 27, 9817-9823. doi: 10.1523/JNEUROSCI.2707-07.2007

Gong, S., Zheng, C., Doughty, M. L., Losos, K., Didkovsky, N., Schambra, U. B., et al. (2003). A gene expression atlas of the central nervous system based on bacterial artificial chromosomes. Nature 425, 917-925. doi: 10.1038/nature02033

Gunaydin, L. A., Yizhar, O., Berndt, A., Sohal, V. S., Deisseroth, K., and Hegemann, P. (2010). Ultrafast optogenetic control. Nat. Neurosci. 13, 387-392. doi: 10.1038/nn.2495

Heimer, L. (1978). "The olfactory cortex and the ventral striatum," in Limbic Mechanisms the Continuing Evolution of the Limbic System Concept, eds K. E. Livingston and O. Hornykiewicz (New York, NY: Plenum), 95-187.

Hikida, T., Kimura, K., Wada, N., Funabiki, K., and Nakanishi, S. (2010). Distinct roles of synaptic transmission in direct and indirect striatal pathways to

\section{FUNDING}

KM was supported by the Narishige Neuroscience Research Foundation, Takeda Science Foundation, Cosmetology Research Foundation, and JSPS KAKENHI Grant Numbers 25830032, 26120709, 16K18377, 16H01671, 17KK0190, and $18 \mathrm{H} 05005$. YF was supported by JSPS KAKENHI Grant Numbers $15 \mathrm{H} 01282$ and 16H04662.

\section{ACKNOWLEDGMENTS}

We thank Drs. Kazuto Kobayashi, Hirohide Takebayashi, and Karl Deisseroth for their generous gift of the plasmids for RNA probes and AAV production. We also thank Hideki Yoshikawa, Eri Murai, Noriko Funabashi, and members of the Fukazawa Laboratory and Life Science Research Laboratory at University of Fukui for technical assistance. This manuscript has been released as a pre-print at bioRxiv (Murata et al., 2018).

reward and aversive behavior. Neuron 66, 896-907. doi: 10.1016/j.neuron.2010. 05.011

Hosoya, Y., and Hirata, Y. (1974). The fine structure of the "dwarf-cell cap" of the olfactory tubercle in the rat's brain. Arch. Histol. Jpn. 36, 407-423. doi: 10.1679/aohc1950.36.407

Ikemoto, S. (2003). Involvement of the olfactory tubercle in cocaine reward: intracranial self-administration studies. J. Neurosci. 23, 9305-9311. doi: 10.1523/JNEUROSCI.23-28-09305.2003

Ikemoto, S. (2007). Dopamine reward circuitry: two projection systems from the ventral midbrain to the nucleus accumbens-olfactory tubercle complex. Brain Res. Rev. 56, 27-78. doi: 10.1016/j.brainresrev.2007.05.004

Inokuchi, K., Imamura, F., Takeuchi, H., Kim, R., Okuno, H., Nishizumi, H., et al. (2017). Nrp2 is sufficient to instruct circuit formation of mitral-cells to mediate odour-induced attractive social responses. Nat. Commun. 8:15977. doi: $10.1038 /$ ncomms 15977

Kobayakawa, K., Kobayakawa, R., Matsumoto, H., Oka, Y., Imai, T., Ikawa, M., et al. (2007). Innate versus learned odour processing in the mouse olfactory bulb. Nature 450, 503-508. doi: 10.1038/nature06281

Kobayashi, K., Sano, H., Kato, S., Kuroda, K., Nakamuta, S., Isa, T., et al. (2016). Survival of corticostriatal neurons by Rho/Rho-kinase signaling pathway. Neurosci. Lett. 630, 45-52. doi: 10.1016/j.neulet.2016.07.020

Liu, Z. H., Shin, R., and Ikemoto, S. (2008). Dual role of medial A10 dopamine neurons in affective encoding. Neuropsychopharmacology 33, 3010-3020. doi: $10.1038 / n p p .2008 .4$

McCutcheon, J. E., Ebner, S. R., Loriaux, A. L., and Roitman, M. F. (2012). Encoding of aversion by dopamine and the nucleus accumbens. Front. Neurosci. 6:137. doi: 10.3389/fnins.2012.00137

Millhouse, O. E., and Heimer, L. (1984). Cell configurations in the olfactory tubercle of the rat. J. Comp. Neurol. 228, 571-597. doi: 10.1002/cne. 902280409

Murata, K., Kanno, M., Ieki, N., Mori, K., and Yamaguchi, M. (2015). Mapping of learned odor-induced motivated behaviors in the mouse olfactory tubercle. J. Neurosci. 35, 10581-10599. doi: 10.1523/JNEUROSCI.007315.2015

Murata, K., Kinoshita, T., Fukazawa, Y., Kobayashi, K., Yamanaka, A., Hikida, T., et al. (2018). Opposing roles of dopamine receptor D1- and D2-expressing neurons in the anteromedial olfactory tubercle in acquisition of place preference in mice. bioRxiv [preprint] doi: 10.1101/421602

Murofushi, W., Mori, K., Murata, K., and Yamaguchi, M. (2018). Functional development of olfactory tubercle domains during weaning period in mice. Sci. Rep. 8:13204. doi: 10.1038/s41598-018-31604-1

Nakamura, T., Karakida, N., Dantsuka, A., Ichii, O., Elewa, Y. H. A., Kon, Y., et al. (2017). Effects of a mixture of medetomidine, midazolam and butorphanol on anesthesia and blood biochemistry and the antagonizing action of 
atipamezole in hamsters. J. Vet. Med. Sci. 79, 1230-1235. doi: 10.1292/jvms. 17-0210

Park, J., Wakabayashi, K. T., Szalkowski, C., and Bhimani, R. V. (2017). Heterogeneous extracellular dopamine regulation in the subregions of the olfactory tubercle. J. Neurochem. 142, 365-377. doi: 10.1111/jnc. 14069

Poulin, J. F., Caronia, G., Hofer, C., Cui, Q., Helm, B., Ramakrishnan, C., et al. (2018). Mapping projections of molecularly defined dopamine neuron subtypes using intersectional genetic approaches. Nat. Neurosci. 21, 1260-1271. doi: 10.1038/s41593-018-0203-4

Saito, H., Nishizumi, H., Suzuki, S., Matsumoto, H., Ieki, N., Abe, T., et al. (2017). Immobility responses are induced by photoactivation of single glomerular species responsive to fox odour TMT. Nat. Commun. 8:16011. doi: 10.1038/ncomms16011

Sano, H., Yasoshima, Y., Matsushita, N., Kaneko, T., Kohno, K., Pastan, I., et al. (2003). Conditional ablation of striatal neuronal types containing dopamine D2 receptor disturbs coordination of basal ganglia function. J. Neurosci. 23, 9078-9088. doi: 10.1523/JNEUROSCI.23-27-09078.2003

Shepherd, G. M. (2004). The Synaptic Organization of the Brain. New York, NY: Oxford University Press.

Stoof, J. C., and Kebabian, J. W. (1981). Opposing roles for D-1 and D-2 dopamine receptors in efflux of cyclic AMP from rat neostriatum. Nature 294, 366-368. doi: $10.1038 / 294366 \mathrm{a} 0$

Xiong, A., and Wesson, D. W. (2016). Illustrated review of the ventral Striatum's olfactory tubercle. Chem. Senses 41, 549-555. doi: 10.1093/chemse/bjw069
Yamaguchi, M. (2017). Functional sub-circuits of the olfactory system viewed from the olfactory bulb and the olfactory tubercle. Front. Neuroanat. 11:33. doi: 10.3389/fnana.2017.00033

Yung, K. K., Bolam, J. P., Smith, A. D., Hersch, S. M., Ciliax, B. J., and Levey, A. I. (1995). Immunocytochemical localization of D1 and D2 dopamine receptors in the basal ganglia of the rat: light and electron microscopy. Neuroscience 65, 709-730. doi: 10.1016/0306-4522(94)00536-e

Zhang, Z., Liu, Q., Wen, P., Zhang, J., Rao, X., Zhou, Z., et al. (2017a). Activation of the dopaminergic pathway from VTA to the medial olfactory tubercle generates odor-preference and reward. Elife 6:e25423. doi: 10.7554/elife.25423

Zhang, Z., Zhang, H., Wen, P., Zhu, X., Wang, L., Liu, Q., et al. (2017b). Wholebrain mapping of the inputs and outputs of the medial part of the olfactory tubercle. Front. Neural. Circuits 11:52. doi: 10.3389/fncir.2017.00052

Conflict of Interest Statement: The authors declare that the research was conducted in the absence of any commercial or financial relationships that could be construed as a potential conflict of interest.

Copyright (c) 2019 Murata, Kinoshita, Fukazawa, Kobayashi, Yamanaka, Hikida, Manabe and Yamaguchi. This is an open-access article distributed under the terms of the Creative Commons Attribution License (CC BY). The use, distribution or reproduction in other forums is permitted, provided the original author(s) and the copyright owner(s) are credited and that the original publication in this journal is cited, in accordance with accepted academic practice. No use, distribution or reproduction is permitted which does not comply with these terms. 